\title{
Management of Primary Failures Related to Fixed Metal Ceramic Bridge Prosthesis Made by Dental Students
}

\section{Debbabi Imen ${ }^{1 *}$, Saafi Jilani ${ }^{2}$, Ben Moussa Anissa ${ }^{2}$, Troudi Fethi ${ }^{3}$ and Cherif Mounir ${ }^{4}$}

${ }^{1}$ DDM, Department of Fixed Prosthodontics, Faculty of Dental Medicine, Tunisia

${ }^{2}$ Professor, Department of Fixed Prosthodontics, Faculty of Dental Medicine, Tunisia

${ }^{3}$ Departement of Laboratory Technician, Hospital University Center of Dental Medicine Monastir, Tunisia

${ }^{4}$ Professor and Head of Department, Department of Fixed Prosthodontics, Faculty of Dental Medicine, Tunisia

*Corresponding Author: Debbabi Imen, DDM, Department of Fixed Prosthodontics, Faculty of Dental Medicine, Monastir, Tunisia.

Received: October 31, 2019; Published: November 11, 2019

DOI: $10.31080 /$ ASDS.2019.03.0699

\begin{abstract}
This case report highlights an esthetic replacement of defective metal ceramic bridge by a ceramic zirconia one after performing the necessary changes in preparations to ensure esthetic and function.
\end{abstract}

Keywords: Prosthetic Dentistry; Esthetic Smile; Zirconia; CAD/CAM; Ceramic Bridge.

\section{Introduction}

Prosthetic dentistry aims to ensure beauty of the smile as well as the harmony of the stomatognathic system. The Common failure of metal ceramic bridge made by student occur during root canal preparation of abutment teeth [1].

Primary complications related to fixed prostheses was a root perforation during preparation for post, a root was fractured during cementation of the post. Vital preparation fractures during try-in or cementation of fixed bridge, oral mucosa was injured during the preparation [2].

The primary failure is more likely due to a lack of skills, which provide predictable function, aesthetics and value for money. Restoration design, clinical execution and patient factors are the most frequent reasons for premature problems [3].

Case presentation

A 32 year old healthy female patient was reported to the department of fixed prosthodontics at the Dental Clinic of Monastir (Tunisia) to remake a bridge replacing the missing teeth (maxillary left first and second premolar) with chief complaint was non- aesthetic smile and over occlusion. Dental history did not reveal any past present symptom.

An intra-oral examination showed an over contoured bridge with supra gingival finish Line that seemed like a gingival recession closed to the canine abutment which caused a discrepancy in the length of the canine with the neighbors naturel and prosthetic teeth. The static occlusion examination highlighted an over occlusion related to the bridge (Figure 1, 2 and 3).

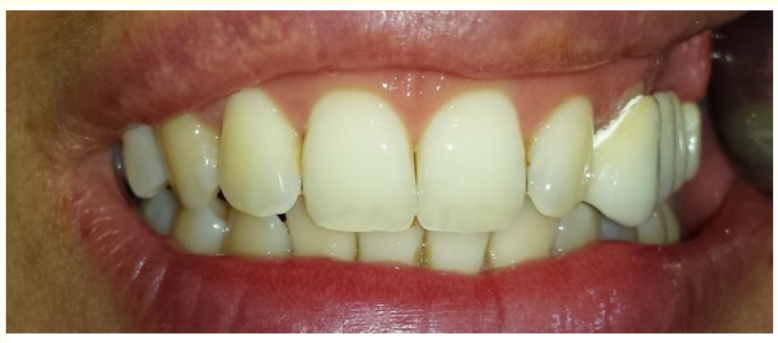

Figure 1: An over contoured bridge with supra gingival finish Line. 


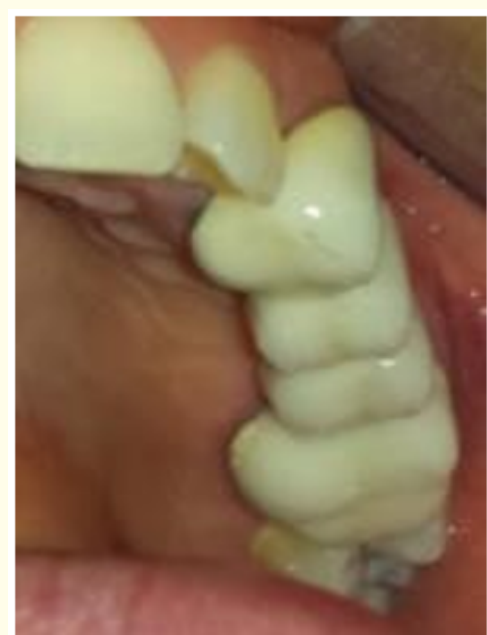

Figure 2: An over contoured metal-ceramic bridge.

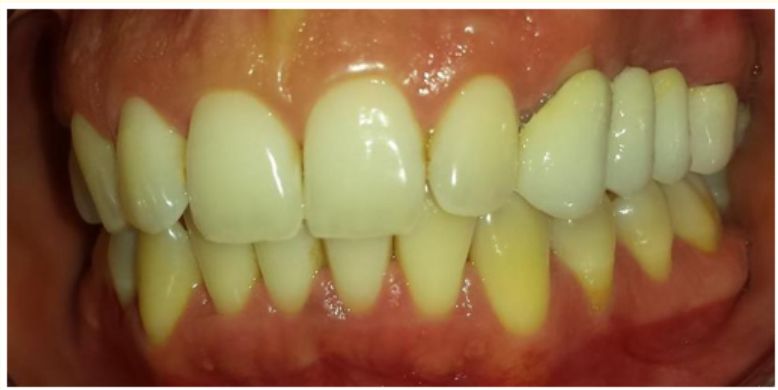

Figure 3: An over occlusion related to the bridge.

After discussion with the patient who sleeked for natural smile and free metal prosthesis, an esthetic correction with ceramic zirconia bridge was planned. The documentation of the bridge was performed with a crown remover and great care was taken to avoid fracture of abutment (Figure 4 and 5).

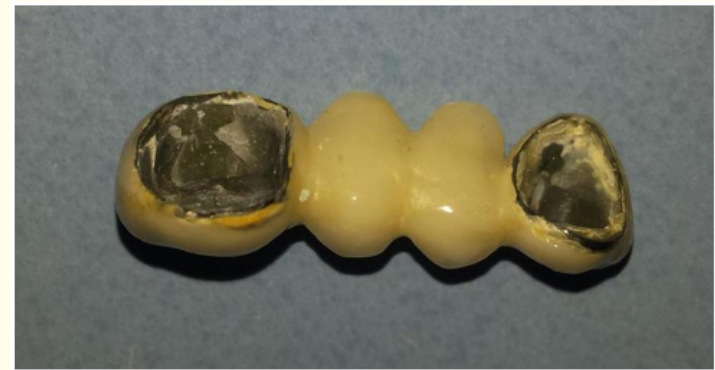

Figure 4: The decementation of the defective metal-ceramic bridge was performed.

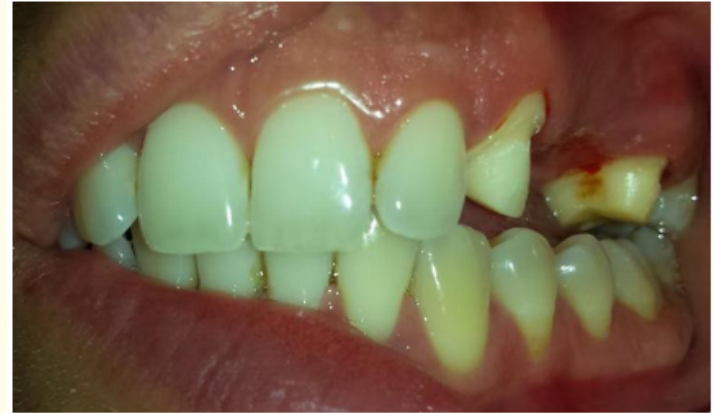

Figure 5: A supra gingival finish line on the canine $(\neq 23)$.

After modification of preparation by cervical relocation of the finish line in subgingival level to hire the margin beneath the gingiva, a temporary bridge was carried out thanks to an index already made in the mouth before removing the old bridge (Figure $6)$. The gingival retraction was carried out using an impregnated cord (23\% aluminum chloride) (Figure 7) and an impression was taken with polyvinyl siloxane material (Virtual, Ivoclar Vivadent) and sent to the laboratory.

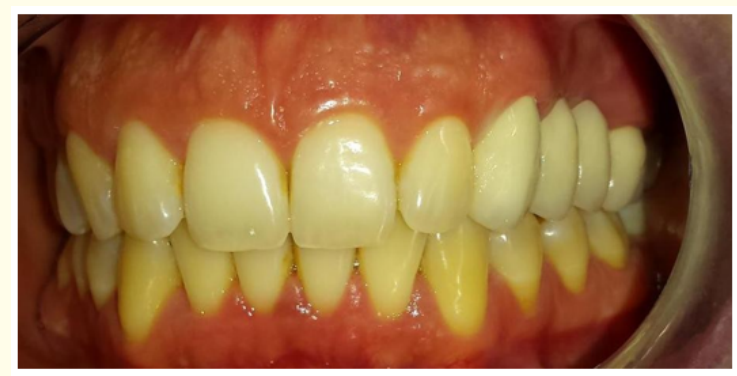

Figure 6: The interim bridge was cemented using a free eugenol cement.

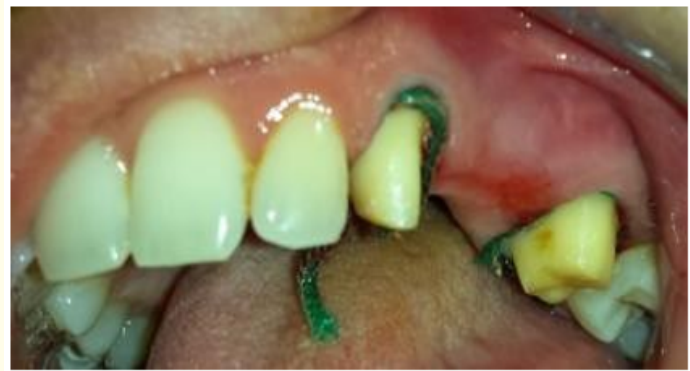

Figure 7: The gingival retraction was carried out using an impregnated cord ( $23 \%$ aluminum chloride). 
Occlusion registration was carried out in centric relation to ensure an accurate functional occlusion and to minimize occlusion corrections. In the clinic, the shade selection was taken with shade guide Vitapan 3 D Master-Vita. Once the working impression is validated, the dental laboratory processed the zirconia framework by CAD/CAM system. After trying the zirconia framework in mouth, the bisque was performed in the lab and tried in the mouth to check esthetics, shade, marginal fit, proximal contacts and static and dynamic occlusion (Figure 8). The adhesive cementation was programmed in the following appointment after staining and glazing.

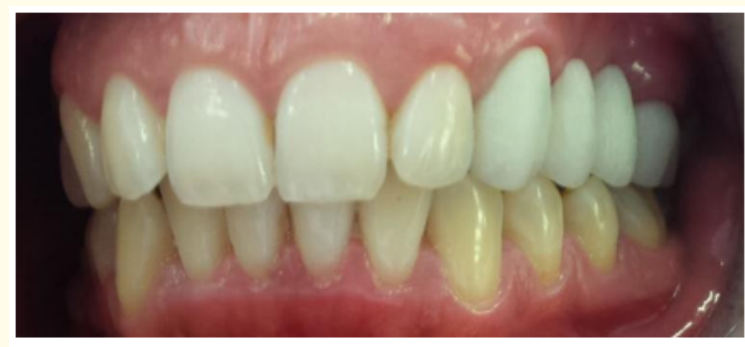

Figure 8A: The static occlusion.

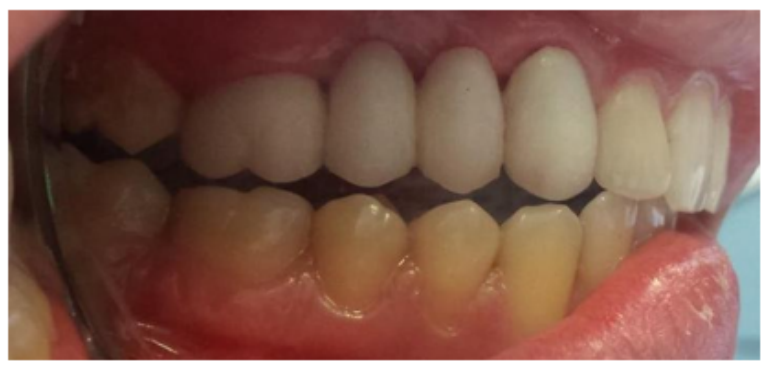

Figure 8B: Dynamic occlusion.

Figure 8A and 8B: The bridge was trying in a bisque state.

The next visit, zircona ceramic Bridge was tried again to verify esthetic, occlusion relationship and than cemented using panavia ${ }^{\text {TM }}$ V5 (Figure 9).

Regular recalls were programmed during which oral hygiene maintenance and gingival margins stability were evaluated. The patient was satisfied with both esthetic and functional outcome (Figure 10).

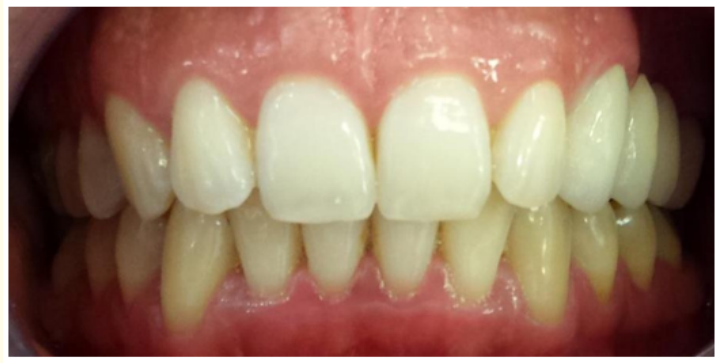

Figure 9: Zirconia bridge was cemented.

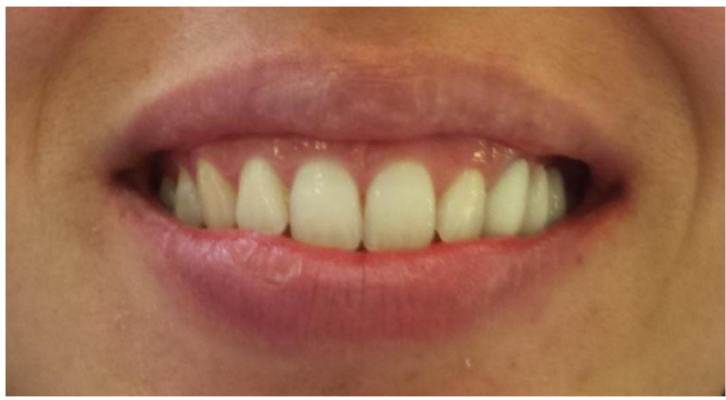

Figure 10: Extra-oral view after cementation with Panavia ${ }^{\mathrm{TM}} \mathrm{v} 5$.

\section{Discussion}

The early failure of a prosthesis is more likely due to biological, esthetic or occlusal factors.

Pulpal and endodontic problems underlying well-functioning crowns and bridges are common. This means that dentists will often be required to undertake endodontic access through existing crowns or bridges or after they have been removed [4-6].

In the presented case the early failure was due to lake of technical skills (supragingival finish line in esthetic zone, over contoured bridge and over occlusion).

Although a little over occlusion could be managed by a simple grinding of the cosmetic ceramic, the patient asked a life-like teeth appearance with pleasant without displaying the margin of the prosthesis, so remaking a new bridge was a legitimate demand. 
Fabricating Zirconia single copings and frameworks involves the manipulation of a 3 - D design on the computer screen, followed by the automated production by a computer -controlled milling machine [7].

Every Zirconia framework alaways requires a try-in step to confirm fit, insertion retention, marginal integrity, and mostly the space left for cosmetic ceramic without omission of the shade appearance. Then, ceramic stratification was performed in the lab $[8,9]$.

The cementation of the brige was performed by panavia ${ }^{\mathrm{TM}}$ V5 which ensure a durable retention. According to Dursun and Col studies, Panavia TM V5, compared to other cements, showed superior adhesive strength [10].

A study conducted by Yogawa and coll on the effect of different primary on the bonding of the zirone using each time the panavia ${ }^{\mathrm{TM}}$ V5 showed that the highest bonding strength is obtained with the Clearfil primer and the version of the Panavia ${ }^{\mathrm{TM}}$ V5, which all contain the functional monomer MDP [11].

During clinic training the students should have always their supervisors in terms of what needs to be done once an imperfection was happened.

Pre-clinical and clinical" training need to be implemented and enforced to improve student's theoretical and practical knowledges. The use of Handouts and e-learning program improved performance of the students' diagnostic and practices skills.

\section{Conclusion}

Dealing with failing indirect restorations is a challenge. When a new bridge was remade, it should satisfy the esthetic and functional demands of patient.

\section{Bibliography}

1. Raustia AM., et al. "Complications and primary failures related to fixed metal ceramic bridge prostheses made by dental students". Journal of Oral Rehabilitation 25.9 (1998): 677-680.

2. Cronström R., et al. "The Swedish patient insurance scheme and guarantee insurance for prosthodontic treatment". International Dental Journal 42.2 (1992): 113-117.
3. Peter Briggs Arijit Ray-Chaudhuri and Kewal Shah. Avoiding and Managing the Failure of Conventional Crowns and Bridges Restorative". Dentistry 78-79.

4. Holm C., et al. "Longevity and quality of FPDs: a retrospective study of restorations 30,20 , and 10 years after insertion". The International Journal of Prosthodontics 16 (2003): 283-289.

5. Tan K., et al. "A systematic review of the survival and complication rates of fixed partial dentures (FPDs) after an observation period of at least 5 years III. Conventional FPDs". Clinical Oral Implants Research 15 (2004): 654-656.

6. Bergenholtz $\mathrm{G}$ and Nyman S. "Endodontic complications following periodontal and prosthetic treatment of patients with advanced periodontal disease". Journal of Periodontology 55 (1984): 63-68.

7. Tinschert J., et al. "Status of current CAD/CAM technology in dental medicine". International Journal of Computerized Dentistry 7 (2004): 25-45.

8. Anissa BM., et al. "Zirconia Based crowns for esthetic rehabilitation of severely discolored Teeth: A case Report". Journal of Oral Health and Dental Science 1 (2017): 1-4.

9. Saafi J., et al. "Esthetic Restoration of Two Severely Decayed maxillary central incisors: a case report". Journal of Oral Health and Dental Science 1.1 (2017): 1-6.

10. Dursun E., et al. "V5: in vitro evaluation project of adhesion to zirconia". biomaterials research unit innovation and interfaces (2015).

11. Yagawa S., et al. "Effect of priming agents on shear bond strengths of resin-based luting agents to a translucent zirconia material". Journal of Prosthodontic Research 62.2 (2018): 204-209.

\section{Volume 3 Issue 12 December 2019} (c) All rights are reserved by Debbabi Imen., et al. 